\title{
Tuberculosis as a Mimicker of Brain Metastasis
}

\author{
Guilherme Wertheimer ${ }^{1}$, Guilherme Rossi Assis-Mendonça ${ }^{1}$, Luciano Souza Queiroz ${ }^{1}$, and \\ Fabiano Reis ${ }^{1}$
}

${ }^{1} \mathrm{UNICAMP}$

October 27, 2020

\begin{abstract}
Multifocal lesions with necrotic center and peripheral enhancement can be a diagnostic challenge. Magnetic resonance imaging with advanced sequences, such as perfusion, can be confusing, as some inflammatory lesions can show hyperperfusion, mimicking tumors. We present a case of neurotuberculosis with multifocal lesions which diagnosis was confirmed by brain biopsy.
\end{abstract}

\section{Tuberculosis as a mimicker of brain metastasis}

Guilherme Soares de Oliveira Wertheimer ${ }^{1}$, Guilherme Rossi Assis-Mendonça ${ }^{2}$, Luciano de Souza Queiroz ${ }^{2}$, Fabiano Reis ${ }^{1}$

1- State University of Campinas, Radiology and Medical Imaging Department

2-State University of Campinas, Pathological Anatomy Department

Corresponding author: Guilherme Soares de Oliveira Wertheimer

-Email: sowgui@gmail.com

-Telephone: + $55193521-7029$

-Address: Rua Tessália Vieira de Camargo, 126 - Cidade Universitária Zeferino Vaz. CEP 13083-887 Campinas, SP, Brazil.

Keywords: Infectious diseases; Neurology; Neurosurgery; Oncology

Key Clinical Message:

Neurotuberculosis is a differential diagnosis to be considered in brain multifocal lesions with peripheral enhancement.

A 52-year-old caucasian man presented with headache, progressive left eye sight impairment and 18Kg weight loss in 5 months.

Brain computed tomography (CT) showed multiple ring enhancing lesions with necrotic center with perilesional edema, hydrocephalus and mass effect. Brain magnetic resonance imaging (MRI) demonstrated multiple cortico-subcortical nodular lesions with necrotic center and peripheric enhancement on $\mathrm{T} 1$ weighted image (WI) after contrast; with hypointense rim on T2 WI and no restricted diffusion in the necrotic center (Figure $1 \mathrm{~A}$ and $\mathrm{B})$.

Biopsy showed a granulomatosus lesion with detection of acid-alcohol resistant bacilli (Figure $1 \mathrm{C}$ and D). The patient received treatment for quaternary tuberculosis, with significant clinical improvement. 
Multifocal lesions with necrotic center and peripheral enhancement (ring enhancing pattern) can be a diagnostic challenge. Even advanced sequences, such as perfusion, can be confusing, as tuberculosis lesions can show hyperperfusion, which is most typically seen in multiple metastatic lesions.

Cerebral spinal fluid (CSF) can contribute to the diagnosis, however tuberculomas may not cause CSF modifications, especially those without contact with the ventricular or cortical surface. Thus, biopsy is still the main tool for the definitive diagnosis.

Neurotuberculosis is a differential diagnosis to be considered in multifocal ring enhancing lesions.

Competing interests

The authors declare no competing interest.

Ethical approval

This study was approved by our Institutional Ethics Committee (CAE number

36223320.7.0000.5404).

Funding Information

There was no funding for this submission.

Acknowledgements

None. No funding to declare.

Author Contribution

Guilherme Soares de Oliveira Wertheimer: drafting the article and final revising.

Guilherme Rossi Assis-Mendonça: histological evaluation and final revising.

Luciano de Souza Queiroz: histological evaluation and final revising

Fabiano Reis: conceived of the presented idea; neuroimaging evaluation; drafting the article and final revising.

All authors contributed to the final manuscript.

References

1- Rakesh, G. K., and Kumar, S. 2011. Central nervous system tuberculosis. Neuroimaging Clin N Am 21:795-814.

2- Batra, A., and Rajendra, P. T. 2003. Perfusion magnetic resonance imaging in intracerebral parenchymal tuberculosis: preliminary findings. J Comput Assist Tomogr 27(6):882 - 8 .

Figure 1: A- Axial brain Computed Tomography (CT) with contrast showing multiple ring enhancing lesions with perilesional edema and mass effect in the left cerebellar hemisphere (white arrow). B- Axial brain Magnetic Resonance Imaging (MRI) with a heterogeneous lesion in the left frontal lobe with hypointensity on T2-weighted image (white arrow). C- Brain histology with hematoxylin and eosin staining, 400x magnification, showed epithelioid histiocytes (black arrowheads). D- Ziehl-Nielsen staining, 1000x magnification, revealed scattered bacilli (black arrow). 

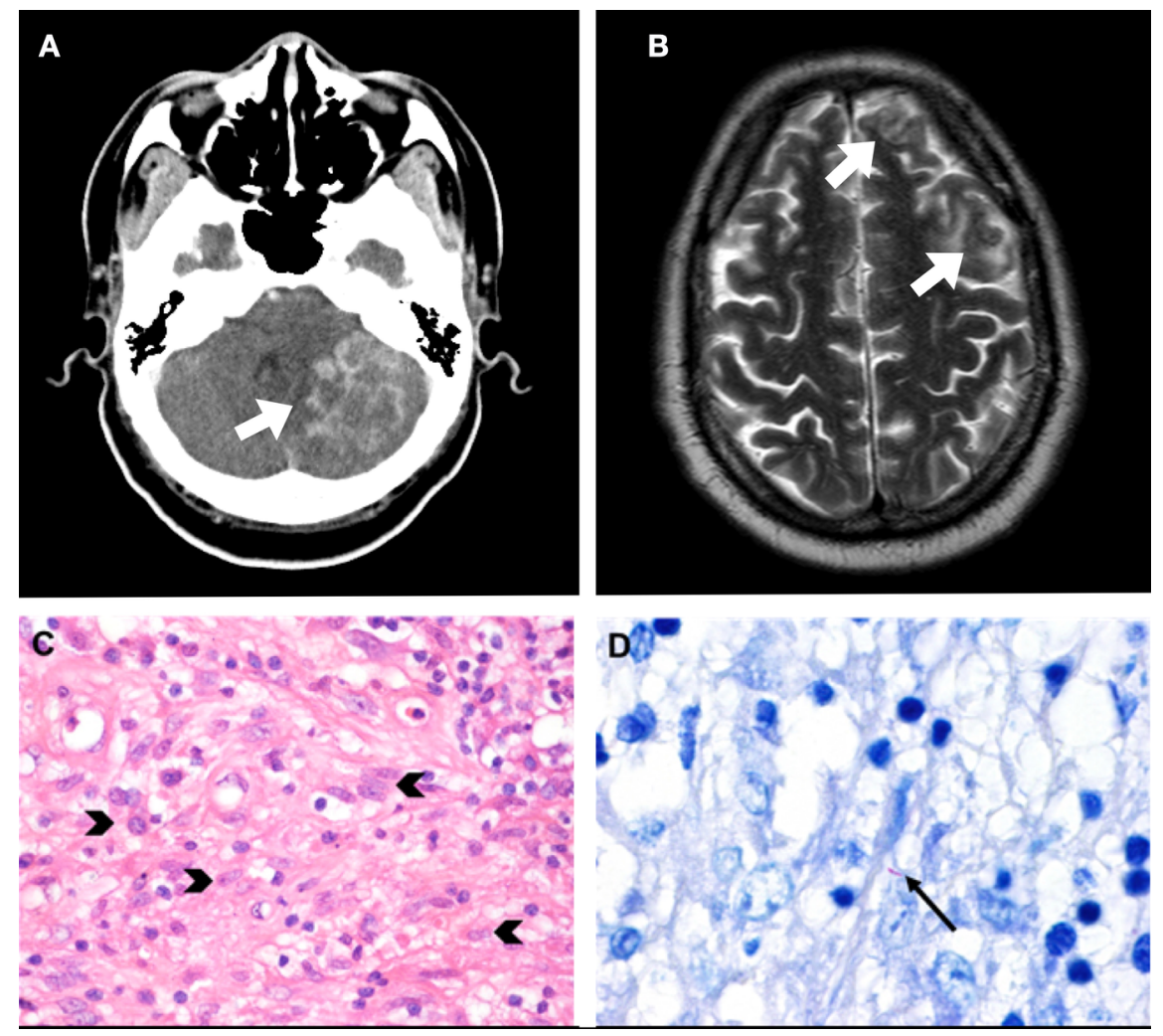